\title{
Motion State Prediction by Unaided Inertial Micro-Machined Accelerometers
}

\author{
Kristoph Keunecke, Gerd Scholl \\ Electrical Measurement Engineering \\ Helmut Schmidt University / University of the Federal Armed Forces, Hamburg, Germany \\ kristoph.keunecke@hsu-hh.de
}

\begin{abstract}
:
Estimating motion velocity state is crucial in dealing with inertial navigation systems (INS) or in situations where backing of an INS by other technologies is difficult or impossible. This study therefore investigates time sequences delivered by embedded inertial sensors in order to draw conclusions about the motion state of moving objects. Various probability tests were evaluated by a simple but typical measurement setup to assess robustness against random walk fluctuations and behavior in the constant velocity state, in order to detect transition from standstill to motion and vice versa. Our investigations end with a proposal for advanced motion state estimation algorithms, where different statistical approaches have been combined.
\end{abstract}

Key words: accelerometer, indoor localization, inertial navigation system, random walk, zero-velocity update.

\section{Introduction}

The satellite based global positioning system (GPS) has certainly revolutionized our everyday lives. However, electromagnetic waves are strongly shielded by the outer shell and walls of buildings. Even if the electromagnetic waves can penetrate into a building, reflection and diffraction effects makes localization and positioning based on electromagnetic wave propagation nearly impossible. Hence, there is still a strong demand for indoor navigation systems, e.g. inertial navigation systems (INS).

Due to enormous development steps in performance, size and price during the last decade many INS today are based on microelectromechanical systems (MEMS). The output of a MEMS sensor typically represents the acceleration or the angular rate of the moving object. Consequently, if one is interested in the position of the moving object, the sensor signal has generally to be integrated twice with respect to time [1]. One of the most challenging tasks in the development of MEMS based INS must surely be to cope with different types of deterministic and stochastic error sources [2][3], e.g. random offset variations, also known as Brownian motion or random walk, infiltrating MEMS at the proof mass by random drifts of molecules [4][5]. Regardless of how random signals are modeled [6][7][8], mastering random fluctuations is still a very challenging task in designing advanced inertial positioning/tracking solutions, even if only the motion state is of interest. Motion state estimation can essentially be subdivided into the seemingly simple tasks of reliably detecting (i) standstill, (ii) transition from zero velocity to moving state, (iii) the unfailing detection of ongoing motion, and (iv) transition from moving to standstill.

This article will describe various approaches with the aim of determining the motion state of an object equipped with strapdown INS. Insight into the demands of unaided inertial positioning is delivered in Section 2. In Section 3 various probability tests are discussed. Measurements to characterize their performance are presented in Section 4. Combining probability tests improves estimation results substantially. This will be demonstrated in Section 5. Concluding remarks are given in the final Section 6.

\section{Requirements concerning unaided INS positioning}

We will start our investigations with an example that is illustrated in Fig. 1. The upper curve in Fig. 1 shows the output $a_{\mathrm{y}}(t)$ of an acceleration sensor, which was mounted on an industrial robot. From standstill the sensor was accelerated with a nearly constant value. At $2 \mathrm{~s}$ 

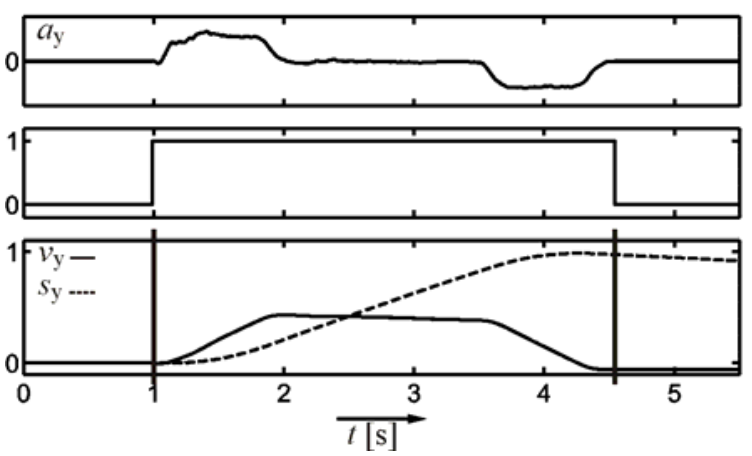

Fig. 1. Remaining of final velocity in spite of effective standstill.

the accelerated motion enters a phase with constant velocity. At about $3.5 \mathrm{~s}$ the sensor is retarded with the same constant absolute acceleration value but opposite sign until motion comes to a standstill at about $4.5 \mathrm{~s}$. The middle curve of Fig. 1 depicts motion states of the sensor. Here ' 0 ' represents standstill and a ' 1 ' denotes motion, independent of the exact value of velocity or acceleration. The lower curve of Fig. 1 displays velocity $v_{y}(t)$ and sensor position $s_{y}(t)$. Vertical lines at $1 \mathrm{~s}$ and $4.5 \mathrm{~s}$ represent transitions between the different motion states 0 and 1 , and vice versa. The short-term movement continuing for $5 \mathrm{~s}$ results in a final velocity of approximately $-0.06 \mathrm{~m} / \mathrm{s}$, a value close to zero, but not identical to zero, thus resulting in a position error of $3.6 \mathrm{~m}$ per minute which continues growing linearly with time, although there is no motion. Clearly, the measurement signal is affected by vibrations, shocks, temperature and Brownian motion, so that acceleration and, accordingly, deceleration regimes are different in size, yielding a velocity unequal to zero. Establishing a threshold velocity [9] in terms of a tolerance band around zero velocity can result in significant positioning errors. Typically, specialized test routines have to be designed for the target applications, e.g. for personal navigation systems operating on gait analysis [10][11]. In [12] and [13] zerovelocity updates are employed based respectively on likelihood tests and hidden Markov models. To reduce position errors, calibration intervals are used for zero-velocity determination. For our universal example this means that if it is possible to determine motion state, i.e. standstill, the integration routine of the navigation/positioning algorithm can be stopped and thus error integration during standstill can be avoided.

\section{Tests}

In the following, let $x(n)$ be the observables of a stationary discrete random sequence $X(n)$ with finite support, i.e. $1 \leq n \leq N$, whose statistical properties do not evolve over time,

$$
\bar{x}=\frac{1}{N} \sum_{\mathrm{k}=1}^{N} x_{\mathrm{k}}=\widehat{\mu}
$$

and

$$
s^{2}=\frac{1}{N-1} \sum_{\mathrm{k}=1}^{N}\left(x_{\mathrm{k}}-\bar{x}\right)=\widehat{\sigma}^{2}
$$

denote the unbiased estimation for the expectation $\mu$ and variance $\sigma^{2}$, respectively, where $x_{\mathrm{k}}$ describes observation at position $\mathrm{k}$.

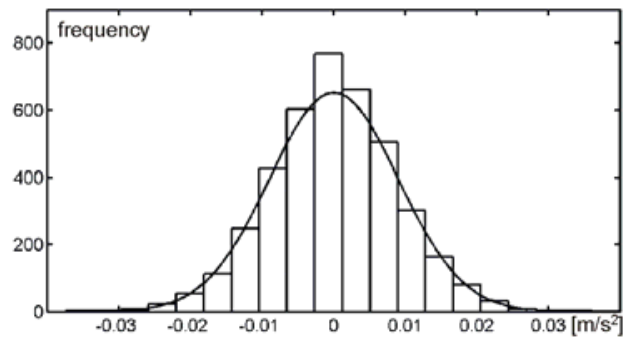

Fig. 2. Approximating a histogram built by raw acceleration data lasting for $4 \mathrm{~s}$ at a standstill by means of a Gaussian distribution function.

With reference to our simple example, during standstill and during a uniform movement without acceleration sensor output should equal zero, but random processes generate a probability density function that is shown in Fig. 2. Data were taken over a time interval lasting for $4 \mathrm{~s}$ with a sampling rate of one sample per ms. The histogram can be approximated by a Gaussian distribution function for which the standard deviation was evaluated as $\widehat{\sigma}=s=0.0093 \mathrm{~m} / \mathrm{s}^{2}$.

Generally, the INS data is interpreted by statistical inference, i.e. hypothesis testing, where in our case the null hypothesis $H_{0}$ represents standstill and $H_{1}$ stands for a moving sensor or object [14]. In the classical approach a test statistic is derived from the measured data and compared with a predefined critical value $(\mathrm{CV})$ on the basis of the significance level $\alpha$, where $\alpha$ is often chosen as e.g. $5 \%$. In our case $\mathrm{CV}$ is determined in a calibration phase before the navigation process starts. If the routine decides on motion, the navigation algorithm will be used otherwise the bias of the INS will be compensated for. A Type I error occurs if $H_{0}$ is rejected even if $H_{0}$ is in fact true. A Type II error occurs if a decision is made for standstill $\left(H_{0}\right)$ even if the alternative hypothesis $H_{1}$ were true. Clearly, Type II errors cause major deviations from the true position. Segmentation is a popular technique in timeseries analysis for estimating $H_{0}$ and $H_{1}$. 
Therefore a subset of $N$ observations of a sensor signal will be examined, generally. In the following we present

- several normality tests and

- $\quad$ one time-based approach

to predict the motion state of unaided accelerometers.

\section{Grubbs test for outliers (GR)}

Typically, one or more samples stand out of the crowd, indicating a starting movement. These samples are commonly called outliers [15]. With the well known transformation

$$
T=\frac{X-\mu}{\sigma}
$$

the probability density function of the random variable $X$ is normalized to the standard normal distribution, where $\mu$ has to be estimated by $\bar{x}$ and $\sigma$ by $s$ resulting in a loss of 2 degrees of freedom in the test statistics. To determine whether an element $x_{\mathrm{k}}$ is an outlier, the test statistic has to be compared with a critical value

$$
C V=\frac{N-1}{N} \sqrt{\frac{t_{\widetilde{\alpha}, N-2}^{2}}{N-2+\mathrm{t}_{\widetilde{\alpha}, N-2}^{2}}}
$$

in which $t_{\widetilde{\alpha}, N-2}^{2}$ is derived from the $t$-distribution with $N-2$ degrees of freedom and $\tilde{\alpha}$ indicates the modified significance level $\alpha / 2 N$. If

$$
\frac{\left|x_{\mathrm{k}}-\bar{x}\right|}{s}>\mathrm{CV}
$$

is true, then $x_{\mathrm{k}}$ will be rejected [16]. In our algorithm we first determine $\bar{x}$ and $\mathrm{s}$ from all $N$ observations and test whether Eq. 3 is true. If this is the case, $\bar{x}$ and $s$ will be recalculated with $N-1$ sample points and the test will be carried out again. This procedure is performed consecutively for all sample points until no further outliers can be detected. If there is more than one outlier, we decide on motion, otherwise standstill is assumed. GR is the sole test routine investigated in this article, which does not need a calibration routine.

\section{Kolmogorov-Smirnov (KS) normality test}

There is a large number of statistical tests dealing with the evaluation of supposed normality for a given data set, e.g. KolmogorovSmirnov. Assume, that the elements of $x(n)$ are arranged in ascending order and follow the presumed Gaussian cumulative distribution function $F(x)$, where $F(x)$ is a theoretical quantity that can be estimated in terms of data using the empirical distribution function

$$
F_{\mathrm{N}}(x)=\frac{n_{\mathrm{Obs}}-x}{N} \text {. }
$$

Here $n_{\text {Obs }} \leq x$ describes the number of observations that are smaller than or equal to $x$ [17][18]. The vertical distance between $F(x)$ and $F_{\mathrm{N}}(x)$ can now be used to introduce a statistical measure. In the case of KS, the test statistic is given by the maximum distance

$$
D=\max \left(D^{+}, D^{-}\right)
$$

between $F(x)$ and $F_{N}(x)$ [19], i.e. where $D^{+}$ denotes the supremum of the magnitude $F_{\mathrm{N}}(x)-F(x)$, if $F_{\mathrm{N}}(x) \geq \mathrm{F}(\mathrm{x})$; and $D^{-}$stands for the supremum of magnitude when $F_{\mathrm{N}}(x)<F(x)$, respectively.

\section{Shapiro-Wilk (SW) normality test}

SW defined another normality test statistic

$$
\mathrm{W}=\frac{\left(\sum_{\mathrm{k}=1}^{N} \zeta_{\mathrm{k}} x_{\mathrm{k}}\right)^{2}}{\sum_{\mathrm{k}=1}^{N}\left(x_{\mathrm{k}}-\mu\right)^{2}}
$$

according to [20][21]. $\zeta_{\mathrm{k}}$ denotes weights, which depend only on the interval length of input data. Thus $\mathrm{W}$ is the quotient of an estimated variance in the numerator that is computed by means of the slope in a quantile-quantile plot, in which the samples of $x(n)$ are compared with the order statistics of a normally distributed set, and sample variance in the denumerator. Here again, $\mu$ is estimated by the sample mean $\bar{x}$. For a Gaussian entity $W$ converges to its maximum, 1, otherwise it tends towards the lower bound, 0 .

\section{Sample variance (S2) of a time-series}

Fluctuations in noise level due to movement or variations of environmental conditions often result in an increased statistical spread $s$ of $x(n)$. These deviations can directly be detected in recorded time-series using second order statistics

$$
s^{2}=E\left\{x_{\mathrm{k}}-\mu\right\},
$$

where $E\{\cdot\}$ denotes the statistical expectation.

\section{$4 \quad$ Test evaluation}

The measurement setup consists of (i) a threeaxis MEMS low-cost inertial sensor [22], which is (ii) integrated into a radio system [23] transferring raw acceleration data to a (iii) control and computing environment.

In this experiment the acceleration sensor was mounted on a carousel, a wooden plate with a diameter of $2 \mathrm{~m}$, mounted on a roller bearing. The carousel was rotated by hand. After an initial push the system slows down due to internal friction losses until it comes to a complete stop. During the first few seconds there is a strong sensor signal, which then slowly fades away until the signal finally falls 
below the sensitivity limit. This is shown in the upper curve of Fig. 3. Sensor path length during motion $s$, shown in the lower curve of Fig. 3 , can be calculated by

$$
s=\varphi \cdot r,
$$

where $\varphi$ is the rotation angle and $r=1 \mathrm{~m}$ is the distance of the sensor to the centre of the carousel. Hence, the total path length of the sensor during motion is $s_{\text {tot }}=41.58 \mathrm{~m}$, corresponding to 6.62 turns of the carousel.

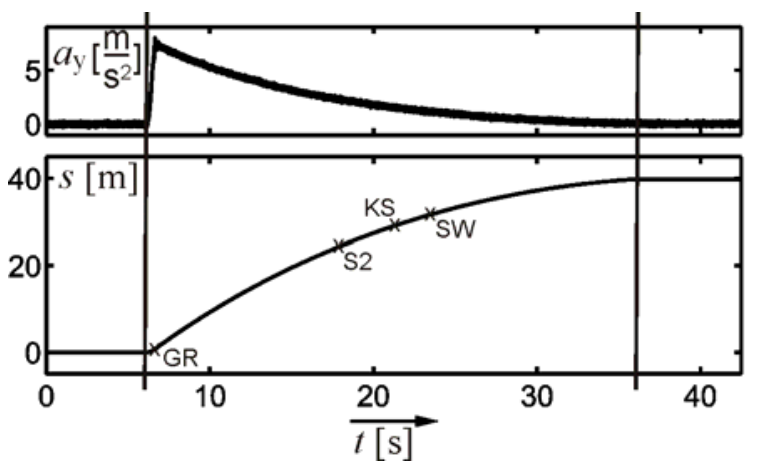

Fig. 3. Raw acceleration data, real movement decision behavior and navigation results with used algorithms.

In the lower curve of Fig. 3 the acronyms indicate the moments when the corresponding probability tests decided on standstill, i.e. when a Type II error occurred for the first time. We thus define a navigation error $s_{\text {err }}$ as the difference between total path length $s_{\text {tot }}$ and the path length that can be associated with the moment when the Type II error occurred.

Shortly after the carousel was set in motion GR $\left(s_{\text {err }}=40.54 \mathrm{~m}\right)$ decided on standstill as the outlier could no longer be detected. S2 type II error occurs approximately after half the motion time equivalent to $s_{\text {err }}=11.41 \mathrm{~m}$. In our experiment $\mathrm{KS}$ delivers $s_{\text {err }}=7.88 \mathrm{~m}$, whereas SW exhibits $s_{\text {err }}=5.67 \mathrm{~m}$. As discussed in the literature [24][25] SW generally has the highest discriminatory power when deviations from normality has to be detected. As expected, the more the sensor signal resembles the sensor signal during standstill, the higher is the error decision rate. This is also documented in Fig. 4.

The stability against random walk fluctuations in standstill can be seen starting from $36 \mathrm{~s}$. Here only inherent sensor noise is detected at the sensor output. By defining a decision error rate (DER) the behavior of the introduced probability tests in standstill is now carried out. With DER we mean the number of incorrect decisions divided by the total number of decisions during the time period of observation.

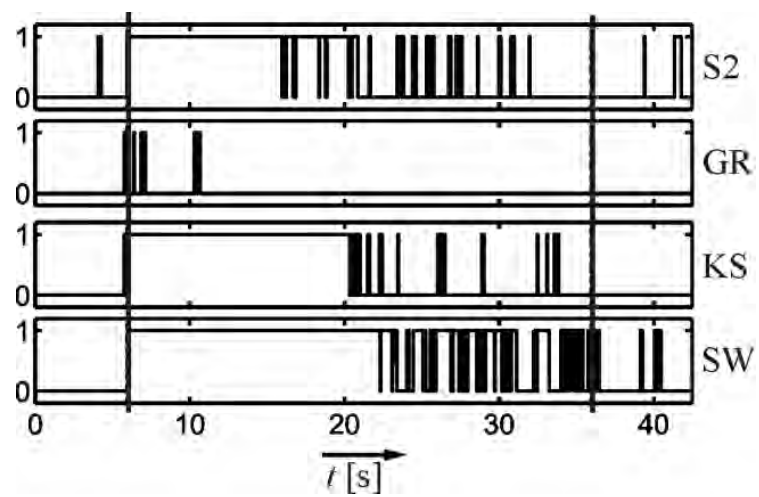

Fig. 4. Decision behavior of used algorithms.

It can be seen that with exception of GR all the tests deliver statistically incorrect decisions. The decision behavior of SW and KS is characterized by only a few sharp peaks, whereas the time periods for wrong decisions are larger for S2 yielding weaker DERs in standstill compared to other approaches. With long-term standstill measurements GR gives the best performance with a DER of approximately $1.5 \cdot 10^{-3}$. KS (DER $=10^{-2}$ ) shows marginal advantages compared to SW $\left(\mathrm{DER}=3 \cdot 10^{-2}\right)$. S2 delivers a DER of roughly $10 \%$ in standstill and is furthermore affected by temperature variations during the measurement.

\section{$5 \quad$ Modified motion prediction approach}

Based on the contributions made in [26] and our presented measurement results it was shown that one single approach will definitely not fulfill all the necessary requirements for highly accurate positioning tasks, i.e. a low DER in standstill, unambiguous detection of transition from standstill to motion and vice versa, and a high reliability in motion recognition, especially when the sensors have to operate close to or below the sensitivity limit. Combinations of probability tests are often a viable solution. In what follows we present GRSW, where we combined two tests based on normality analysis, i.e. GR and SW.

Here, GR is best suited for finding outliers and thus finding the exact moment of a transition from standstill to motion. If SW indicates a transition at the same moment, reliability of estimation can be improved significantly. Fig. 5 presents an example, in which a transition from standstill to motion is clearly indicated at about $5 \mathrm{~s}$. 


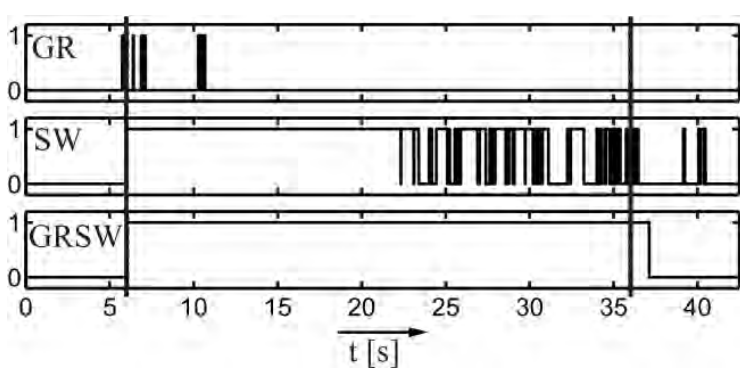

Fig. 5. Decision behavior of stand-alone $G R$ and SW and modified approach GRSW.

Incorrect decisions are made frequently by SW in the time interval starting at $23 \mathrm{~s}$, where the sensor signal insidiously approaches the sensitivity limit. Comparing the true motion and standstill states of sensor unit shows that the DER of SW in motion state is significantly higher than in standstill, which is due to small non-deterministic, non-Gaussian variations caused by a slight jerking movement of the carousel. This characteristic feature can be used to classify motion states in defining a decision error rate for motion, MDER. Thus, if MDER is significantly higher than the mean DER during the calibration phase, which shall be denoted by $\overline{C D E R}$, the evaluating algorithm decides on the motion state. This means, that a $\mathrm{CV}$ for the motion state can be calculated as

$$
\mathrm{CV}(\mathrm{MDER})=m \cdot \mathrm{DER},
$$

where $m>1$, generally. For our experiment a value of $m=1.1$ delivered best results, which is shown in the lower curve of Fig. 5. With a sample rate of $1 \mathrm{kSamples} / \mathrm{s}$ the decision on standstill is typically made at about $1 \mathrm{~s}$ after the actual stop of motion. But since the angular velocity shortly before the carousel comes to a final stop is very small, path length errors due to a delayed decision are also small.

\section{Conclusion}

In this article various probability tests were evaluated in their performance to detect the motion state of objects equipped with inertial sensors, especially MEMS acceleration sensors. Principally, all tests can be used for detecting a beginning motion, as the observation vector typically shows characteristic variations. It has been shown that none of all the tests under investigation was able to fulfill all the requirements at the same time and thus a combination of probability tests for motion state detection is strongly recommended. Which combination should be chosen depends on the final target application. With GRSW a DER in standstill better than $10^{-4}$ under random fluctuations and variations in noise floor was achieved.

\section{References}

[1] P. G. Savage, Strapdown analytics. Maple Plain, Minnesota: Strapdown Associates Inc., 2007, vol. 2.

[2] A. Lawrence, Modern inertial technology: navigation, guidance, and control, 2nd ed., New York: Springer Verlag, 1998.

[3] D. H. Titterton and J. L. Weston, Strapdown Inertial Navigation Technology, 2nd ed. Stevenage: Institution of Electrical Engineers, 2004.

[4] H. Külah, J. Chae, N. Yazdi, and K. Najali, "Noise Analysis and Characterization of a Sigma-Delta Capacitive Microaccelerometer," IEEE Journal of Solid-State Circuits, vol. 41, no. 2, Feb. 2006.

[5] T. Gabrielson, "Mechanical-thermal noise in micromachined acoustic and vibration sensors," IEEE Transactions on Electron Devices, vol. 40, no. 5, May 1993.

[6] A. Gelb, Applied Optimal Estimation, 1st ed., Massachusetts: The M.I.T.Press, 1974.

[7] P. S. Maybeck, Stochastic Models, Estimation, and Control. New York: Academic Press, 1979, vol. 1.

[8] N. El-Sheimy, H. Hou, and X. Niu, "Analysis and Modeling of Inertial Sensors Using Allan Variance," IEEE Transactions on Instrumentation and Measurement, vol. 57, no. 1, Jan. 2008.

[9] F. Reinboth, "Interne Stützung zur Genauigkeitsverbesserung in der Inertialmesstechnik," Ph. D., Universität Karlsruhe, 2000.

[10] E. Foxlin, "Pedestrian Tracking with ShoeMounted Inertial Sensors," IEEE Computer Graphics and Applications, vol. 25, no. 6, Nov. 2005.

[11] V. Gabaglio, "GPS/INS Integration for Pedestrian Navigation". Ecole polytechnique federale de Lausanne, Ph. D., Lausanne, 2003.

[12] I. Skog, P. Händel, J.-O. Nilsson, and J. Rantakokko, "Zero-Velocity Detection - An Algorithm Evaluation," IEEE Transactions on Biomedical Engineering, vol. 57, no. 11, Nov. 2010.

[13] Y. S. Suh and S. Park, "Pedestrian Inertial Navigation with Gait Phase Detection Assisted Zero Velocity Updating," Proceedings of the 4th International Conference on Autonomous Robots and Agents, Feb. 2009.

[14] R. Parsons, Statistical Analysis: a decision making approach, 2nd ed., New York: Harper \& Row, 1978.

[15] V. Barnett and T. Lewis, Outliers in statistical data, 2nd ed. Chichester: John Wiley \& Sons, 1987.

[16] F. E. Grubbs, "Procedures for Detecting Outlying Observations in Samples," Technometrics, vol. 11, no. 1, Feb. 1969. 
[17] D. A. Darling, "The Kolmogorov-Smirnov, Cramer-von Mises Tests," The Annals of Mathematical Statistics, vol. 28, no. 4, Dec. 57.

[18] R. B. D'Agostino and M. A. Stephens, Goodnessof-fit techniques. New York: Marcel Dekker, 1986.

[19] M. A. Stephens, "EDF Statistics for Goodness-offit," Technical Report No. 186, Office of Naval Research, Stanford University, Stanford, CA, 1972.

[20] S. S. Shapiro and M. B. Wilk, "An Analysis of Variance Test for Normality (Complete Samples)," Biometrika, vol. 52, no. 3/4, Dec. 1965.

[21] J. P. Royston, "A simple method for evaluation the Shapiro-Francia W' Test of Non-Normality," Journal of the Royal Statistical Society. Series D (The Statistician), vol. 32, no. 3, Sep. 1983.

[22] Freescale Semiconductors - Three Axis Low-g Micromachined Accelerometer MMA 7331LC Technical Data, 2010.

[23] H.-J. Körber, H. Wattar, and G. Scholl, "Modular Wireless Real-Time Sensor/Actuator Network for Factory Automation Applications," IEEE Transactions on Industrial Informatics, vol. 3, no. 2, May 2007.

[24] H. C. Thode, Testing for normality. New York: Marcel Dekker, 2002.

[25] S. S. Shapiro, M. B. Wilk, and H. J. Chen, "A comparative study of various tests for normality," Journal of the American Statistical Association, vol. 63, no. 324, 1968.

[26] K. Keunecke, and G. Scholl, "Bestimmung des Bewegungszustandes von Objekten durch Analyse des Rauschverhaltens von StrapdownMEMS-Beschleunigungssensoren", XXVI. Messtechnisches Symposium AHMT, Aachen, Sep. 2012. 